\title{
Sampling Small Volumes of Saliva for Determination of the Stress Hormone $\alpha$-Amylase. A Comparative Methodological Study
}

\author{
Aristidis Arhakis, Vassilis Karagiannis, Sotirios Kalfas \\ School of Dentistry, Aristotle University of Thessaloniki, Thessaloniki, Greece \\ E-mail: oaristidis@gmail.com \\ Received May 5, 2011; revised June 17, 2011; accepted July 5, 2011
}

\begin{abstract}
Salivary alpha amylase was taken into account as an important physiological indicator in psychoneuroendocrinological research focusing on stress. Two sampling devices that allow saliva collection through absorption to a cotton roll (Salivette ${ }^{\circledR}$-method) or to small cotton pellets (VectaSpin ${ }^{\mathrm{TM}}$ Micro [VSM]-method) were studied. Any loss of salivary alpha-amylase (sAA) activity in relation to the saliva volume absorbed and harvested by centrifugation was examined. A pooled saliva sample prepared from stimulated whole saliva (collected by drooling) of 30 subjects was used. Three different saliva volumes $(2.9 \mathrm{ml}, 1.5 \mathrm{ml}$, and $0.8 \mathrm{ml})$ were tested on cotton rolls and two $(0.03 \mathrm{ml}$, and $0.015 \mathrm{ml})$ on cotton pellets. The sample sAA activity was determined from the hydrolysis of 2-chloro-4-nitrophenyl- $\alpha$-D-maltotrioside. In comparison with the original drooling sample, no sAA loss was observed in $1.5 \mathrm{ml}$ samples tested with Salivette, while a significant decrease of activity was recorded with smaller volumes. VSM collected samples showed a non-volume dependent decrease of sAA activity of about $25 \%$. Salivette requires large saliva volumes to allow an accurate sAA estimation. With cases of limited saliva access, VSM may be a suitable sampling device.
\end{abstract}

Keywords: Salivary Alpha Amylase, Enzyme Activity, Method, Saliva Sampling

\section{Introduction}

Saliva is of great clinical significance as a diagnostic fluid and analysis of its components may provide information on local or systemic diseases and disorders [1-5]. Sampling saliva is clinically more advantageous compared to other body fluids since it is performed with noninvasive techniques without the stressful exposure to needles [6-9]. Whole saliva can be sampled by either the draining method, the spitting method, the suction method, or the absorbent (swab) method [10].

A device under the brand name Salivette ${ }^{\circledR}$ (Sarstedt, Numbrecht, Germany) is frequently employed for whole saliva collection by the absorbent method [11]. The components of the devise are a cotton roll, and a plastic centrifuge tube with an inner cylindrical strainer (Figure 1). The cotton roll is placed in the mouth for up to $5 \mathrm{~min}$ to absorb saliva till the roll becomes well soaked (about 3 $\mathrm{ml}$ saliva). The saliva is then harvested by centrifuging the roll in the tube with the strainer.

Salivette ${ }^{\circledR}$ may be difficult or less suitable to use under certain circumstances. Soaking the cotton roll with saliva requires time and the presence of a certain saliva volume in the mouth. To enable saliva collection, many researchers ask the subjects to chew the cotton roll; however, this is unsuitable when unstimulated saliva has to be collected. Occasionally, young children may find the chewing procedure cumbersome and refuse to co-operate. Repeated short interval-sampling may suffer by a limited amount of saliva absorbed to the roll, which could affect the composition of the final sample. Indeed, an error variance in the measurement of cortisol, testosterone, DHEA, estradiol, progesterone, and salivary $\alpha$-amylase (sAA), has been reported, particularly with low sample volumes [1214].

The concentration of sAA in saliva is considered as an indicator of the sympathetic adrenal medullar system activation due to stress [15-17]. Saliva for sAA assessment has been sampled with Salivette ${ }^{\circledR}$ or by the passive drooling technique. However, these techniques may not be feasible when small sample volumes are to be expected, as mentioned above. To overcome this problem, 


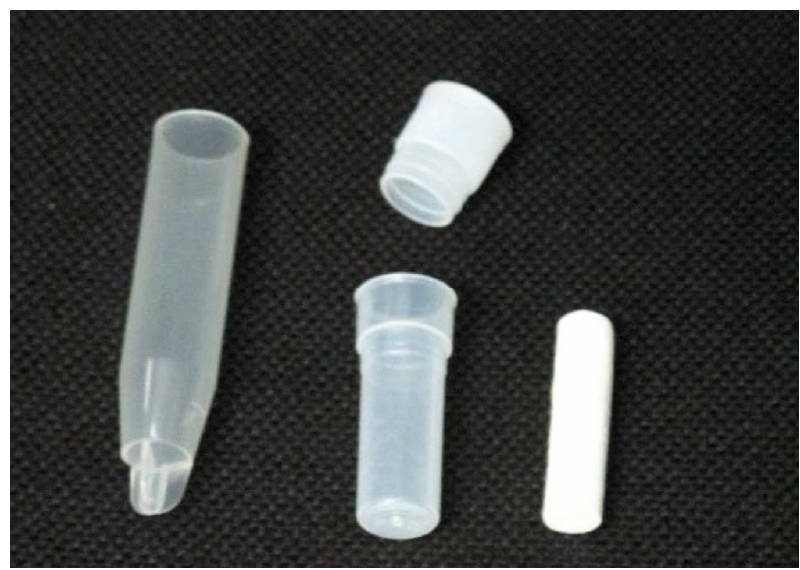

(a)

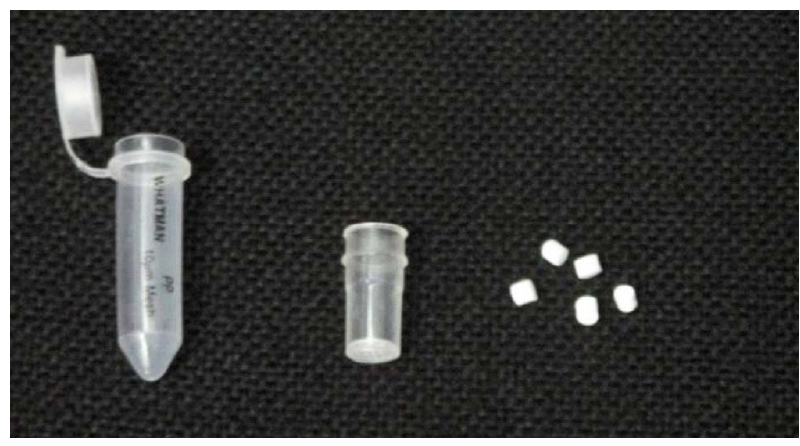

(b)

Figure 1. The sampling devises Salivette ${ }^{\circledR}$ (a) and VectaSpinTM Micro (b) used in the study. From the left to the right, the centrifugation tube, the strainer and the saliva absorbent material (cotton roll or pellets) for each device are seen.

we tried a micro-method that allows sampling of small saliva quantities, using the centrifugation device Vec$\operatorname{taSpin}^{\mathrm{TM}}$ Micro (Whatman, Middlesex, UK) and small cotton pellets for rapid absorption of saliva from the mouth (Figure 1). The suitability of the two absorbent methods for sampling saliva for sAA assessment was evaluated in this study. In particular, any loss of sAA activity in relation to the saliva volume absorbed was examined.

\section{Methods}

\subsection{Saliva Sample}

A pooled whole saliva sample from 30 adults was used throughout the experiment [18]. The subjects were asked to refrain from drinking, eating any food [19] physically exercising [20] and from smoking [21] two hours prior to sampling. They rinsed their mouths once with tap water and started collecting unstimulated saliva, by passive drooling, $15 \mathrm{~min}$ later. The saliva was allowed to drip off the lower lip into a graduated test tube fitted with a funnel as described [10,14] for 15 - $20 \mathrm{~min}$.

From each subject, a saliva sample of approximately 5 $\mathrm{ml}$ was collected. The samples were centrifuged $(10,000$ $\mathrm{x} g, 5 \mathrm{~min})$ to remove particles and kept at $-80^{\circ} \mathrm{C}$ [17]. On the day of the experiment, the samples were thawed and mixed together to obtain the required volume of pooled saliva.

\subsection{Experimental Design}

Saliva from the pooled sample was pipetted to cotton rolls of Salivette ${ }^{\circledR}$ and to cotton pellets used with VectaSpin ${ }^{\mathrm{TM}}$ Micro (VSM). Three different amounts $(2.9 \mathrm{ml}$, $1.5 \mathrm{ml}$, and $0.8 \mathrm{ml}$ ) of saliva were tested on cotton rolls (15 rolls with each volume) and two amounts $(0.03 \mathrm{ml}$, and $0.015 \mathrm{ml})$ on cotton pellets $(150$ pellets with each volume).

Each Salivette cotton roll was placed in the strainer of the centrifuge tube and saliva was carefully pipetted on the top of the roll till the determined volume was applied. At its maximum, a cotton roll can absorb about $3 \mathrm{ml}$. The saliva was harvested from the roll to the bottom of the tube by centrifugation ( $\mathrm{x} \mathrm{g}, 20 \mathrm{~min}$ ).

Each cotton pellet (Roeko etching pellets, medium size, $\varnothing 3 \mathrm{~mm}$, ref 230 002) used in the VSM method can maximally absorb $0.03 \mathrm{ml}$. A volume of $\geq 0.05 \mathrm{ml}$ final sample was required for each measurement. To obtain this volume, 5 cotton pellets were placed in the strainer and the corresponding amount of saliva $(5 \times 0.03 \mathrm{ml}$ or 5 $\times 0.015 \mathrm{ml})$ was applied on them. After centrifugation $(10,000 \times \mathrm{g}, 5 \mathrm{~min})$, the saliva was harvested in the bottom of the tube.

From the pooled saliva, 15 aliquots of $0.01 \mathrm{ml}$ each were aspirated and analyzed for comparative purpose. These samples are referred in the text as the drooling samples. The experiment was accomplished on two occasions, with one third of the samples of each method being examined on the first occasion and the rest of them on the second occasion, 3 weeks later. All saliva samples were kept at $6^{\circ} \mathrm{C}$ until analyzed for sAA activity (within $2 \mathrm{~h}$ ). Immediately before the analysis, each sample was diluted 200 times with distilled water.

\subsection{Measurement of sAA Activity}

The activity of sAA (in U/ml sample) was determined from the hydrolysis of the chromogenic substrate 2-chloro4-nitrophenyl- $\alpha$-D-maltotrioside [22]. A volume of 0.6 $\mathrm{ml}$ freshly prepared substrate solution (5 mM 2-chloro-4nitrophenyl- $\alpha$-D-maltotrioside in reaction buffer) was mixed with $0.05 \mathrm{ml}$ diluted sample in a cuvette. The hydrolysis of the substrate was spectrophotometrically fol- 
lowed at $405 \mathrm{~nm}$, through the production of the coloured compound 2-chloro-p-nitrophenol. The reaction buffer contained $0.03 \%$ bovine albumin, $0.06 \% \mathrm{CaCl}_{2}, 0.48 \%$ $\mathrm{KSCN}$, and $0.03 \% \mathrm{NaN}_{3}$ in $50 \mathrm{mM}$ 2-(N-morpholino) ethanesulfonic acid [23]. The $\mathrm{pH}$ of the buffer was adjusted to 6.0 with $5 \mathrm{M} \mathrm{KOH}$ solution, since, in a preliminary experiment, the sAA activity was found to be higher at $\mathrm{pH} 6.0$ than at $\mathrm{pH} 7.0$.

One $\mathrm{U} / \mathrm{ml}$ of sAA was defined as the amount of enzyme that hydrolyzes $1 \mu \mathrm{mol}$ substrate per min [24]. The molar absorptivity of 2-chloro-p-nitrophenol was found to be $14,6 \mathrm{l} / \mathrm{mol} \cdot \mathrm{cm}$, measured with solutions of pure 2-chloro-p-nitrophenol (concentration range 0.0025 - 0.5 $\mathrm{mM}$ ) under the present experimental conditions. Purified $\alpha$-amylase type XIII (product No. A1031) was used as the positive control. All experiments were done at room temperature. The chemicals were purchased from SigmaAldrich Chemie Gmbh, Switzerland.

\subsection{Statistical Analysis}

Based on the results from a pilot study with 6 samples in each group, a power analysis showed that with $a=0.05$ and $N=90$ (equal size groups), the null hypothesis of the equality of means under a one-way anova model would be rejected with $99 \%$ probability. Accordingly, the sizes of the samples for Salivette, VSM, and drooling methods were determined to be 15,30 , and 15 , respectively.

The assumptions of normality and equality of variances where verified by the Sapiro-Wilk and Levene's Test, respectively. The statistics Min, Max, Mean, SD, and $95 \% \mathrm{CI}$ were used to describe the outcome variable. The main hypothesis of equality of means was evaluated by the one-way Anova model, while pair wise comparisons were conducted by the Tukey's HSD method. The overall analysis was performed with SPSS 15.0 software and the level of statistical significance was set at $p<$ 0.05 .

\section{Results}

The descriptive statistics for the sAA activity of the samples obtained by the three methods are presented in Table 1. The hypothesis concerning the equality of means of the 6 groups were rejected $(F(5,114)=7.094, p<$ 0.001 , one way ANOVA). The results of the pair wise comparisons with Tukey's HSD test are shown in Figure 2. The statistically significant differences in sAA activity are indicated in the same figure. No significant differences were observed between the groups: a) Salivette 2.9 $\mathrm{ml}$ and $1.5 \mathrm{ml}(p=0.582)$, b) Salivette $2.9 \mathrm{ml}$ and drooling $(p=0.979), \mathrm{c})$ Salivette $1.5 \mathrm{ml}$ and VSM 0.03 $\mathrm{ml}(p=0.2), \mathrm{d})$ Salivette $1.5 \mathrm{ml}$ and VSM $0.015 \mathrm{ml}(p=$
Table 1. Descriptive statistics for sAA activity (in $\mathrm{U} / \mathrm{ml}$ ) detected in saliva samples obtained with the three methods.

\begin{tabular}{ccccc}
\hline Method & $\begin{array}{c}\text { Saliva volume } \\
\text { absorbed }(\mathrm{ml})\end{array}$ & $\begin{array}{c}\text { Sample } \\
\text { number }\end{array}$ & Mean \pm SD & $\begin{array}{c}\text { Range } \\
\text { (Min-Max })\end{array}$ \\
\hline \multirow{2}{*}{ Salivette $^{\circledR}$} & 2.9 & 15 & $14.4 \pm 3.4$ & $7.7-22.4$ \\
& 1.5 & 15 & $12.5 \pm 3.2$ & $7.7-17.9$ \\
VSM & 0.8 & 15 & $9.2 \pm 4.0$ & $2.9-14.3$ \\
Drooling & 0.03 & 30 & $10.1 \pm 3.5$ & $3.8-16.4$ \\
& 0.015 & 30 & $10.4 \pm 2.5$ & $4.1-13.6$ \\
\hline
\end{tabular}

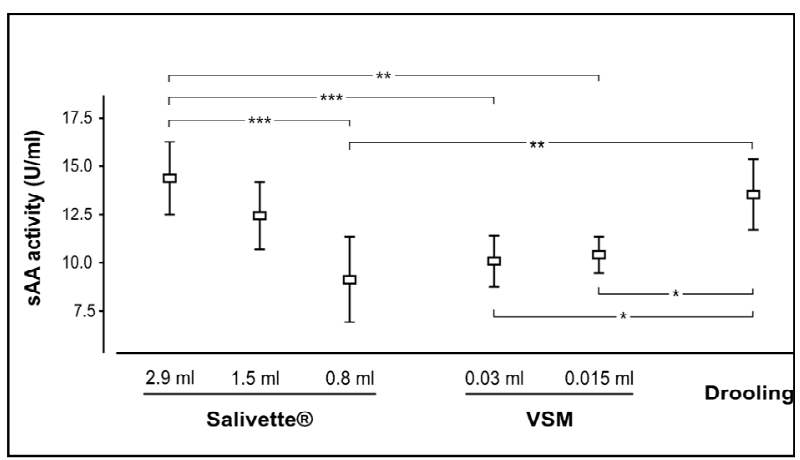

Figure 2. Mean (rectangular) and $95 \%$ Confidence Interval (bar) for SA activity of saliva samples obtained with the three methods. The statistically significant differences are indicated with asterisks $\left(*: p<0.5,{ }^{* *}: p<0.01,{ }^{* * *}: p<\right.$ 0.001).

$0.360)$, e) Salivette $1.5 \mathrm{ml}$ and drooling $(p=0.942), \mathrm{f})$ Salivette $0.8 \mathrm{ml}$ and VSM $0.03 \mathrm{ml}(p=0.943)$, g) Salivette $0.8 \mathrm{ml}$ and VSM $0.015 \mathrm{ml}(p=0.818)$, and h) VSM $0.03 \mathrm{ml}$ and $0.015 \mathrm{ml}(p=0.998)$. The difference between Salivette $1.5 \mathrm{ml}$ and $0.8 \mathrm{ml}$ was marginally nonsignificant $(p=0.067)$.

\section{Discussion}

It is evident from the present results that the saliva volume absorbed by the cotton roll may influence the sAA concentration detected in the final sample. To prevent significant loss of sAA, the cotton roll of Salivette has to be soaked with approximately $\geq 1.5 \mathrm{ml}$ saliva, which equals to about $\geq 50 \%$ of the volume that the roll can maximally absorb. Decreasing the saliva volume to 0.8 $\mathrm{ml} /$ roll resulted in a statistically lower sAA activity in comparison with the other two volumes tested, i.e. 2.9 and $1.5 \mathrm{ml}$, in the Salivette group as well as the sample collected by drooling.

Salivette has been used for sampling saliva in previous studies evaluating stress through sAA activity $[11,17]$. These authors provided no information for the possible 
effect of a partially soaked cotton roll on the estimation of sAA activity, probably, because they did not face the limitation of a small sample volume. Under certain circumstances, it may be difficult to obtain the saliva amount needed for saturation of the roll. Young children may not be capable or even refuge to provide the required volume. Occasionally, the size of the roll may be disproportional to the small mouth of the child, or the procedure itself may seem unbearable or stressful for a subject who must accept the cotton roll in the mouth for several minutes. Furthermore, repeated sampling with short intervals, e.g. $<1 \mathrm{~min}$, is unfeasible, since the flow rate of stimulated saliva is usually $<1.5 \mathrm{ml} / \mathrm{min}$.

The micromethod VSM helps to overcome these disadvantages and it is in fact a modification of the Salivette method. The voluminous cotton roll was replaced by small cotton pellets and the VectaSpin ${ }^{\mathrm{TM}}$ centrifugation devise was used to harvest the sample. VSM allows a more rapid collection, about 2 - 3 seconds per pellet, the procedure being more comfortable for the subject and demanding a minimum of cooperation. Five saliva-soaked pellets provide a sufficient sample volume for sAA analysis.

The sAA activity detected in VSM-collected samples was significantly lower than in the corresponding samples collected by drooling or by Salivette-cotton roll soaked with $\geq 1.5 \mathrm{ml}$. Compared to the drooling sample, a $25 \%$ and $23 \%$ mean loss of sAA activity were found for the volumes 0.03 and $0.015 \mathrm{ml}$, respectively. The corresponding mean values for the Salivette-collected samples of 0.8 and $1.5 \mathrm{ml}$ were $33 \%$ and $7 \%$, respectively. Interestingly, a higher variation was recorded in sAA activity among the Salivette $0.8 \mathrm{ml}$ samples (Coefficient of variation 44\%) than among the VSM samples of 0.03 or $0.015 \mathrm{ml}$ (Coefficient of variation $35 \%$ and $24 \%$, respectively).

Although not proved, the loss of sAA activity is probably due to a partial absorption of the enzyme by the plastic surfaces of the devise and by the cotton. The extent of protein absorption to a surface is influenced by the chemical composition of the protein-containing solution and the physicochemical properties of the surface. The protein composition of saliva varies depending on the serous or mucous character of the secretory gland. It may also vary among different subjects. A high salivary content of proteins competing with sAA absorption prevents extensive loss of sAA and vice versa. To avoid confounding results due to the intra-subject variation, a pooled saliva sample collected from several subjects, was used throughout this study. Under these conditions, the rich protein supply provided by saliva volumes of $\geq 1.5 \mathrm{ml}$, seems to sufficiently prevent sAA loss, probably by blocking the protein binding sites in the cotton roll and tube walls of Salivette. On the contrary, the protein content in the 100 times smaller saliva volume obtained by the VSM method, appears inadequate to inhibit sAA absorption to the devise surfaces. The same phenomenon probably occurs with the $0.8 \mathrm{ml}$ volume in Salivette.

The saliva obtained after centrifugation with Salivette or VSM is less turbid and viscous than the initial fluid. This indicates that the cotton roll and pellets retained particles, such as microbes, epithelial cells, and food debris, that confer turbidity and also macromolecules, such as mucins that are mainly responsible for the high viscosity of saliva. Removal of particles was also achieved by the initial centrifugation step, performed before pooling the individual saliva samples together. The clarified and less viscous saliva obtained by centrifugation and filtration may, occasionally, be advantageous for the following handling and analysis steps. However, these procedures may also affect the compound under test. Removal of microbes also removes sAA bound to the cell surface of certain oral bacterial species [25]. On the other hand, the cotton roll of Salivette seems to retain most of salivary mucins but not $\mathrm{sAA}$ when adequately soaked with saliva.

To conclude, the use of Salivette for collecting saliva samples yields no loss in sAA activity, provided that the sample volume is $\geq 1.5 \mathrm{ml}$. When the available saliva volume is small $(<1 \mathrm{ml})$, Salivette cannot be used, however, the micromethod VSM described above may provide a good estimation of sAA activity, the loss being about $20 \%$ in the volume range $0.03-0.015 \mathrm{ml}$. Since sAA is a potential indicator of autonomic response to stress a reliable sampling method is required. These findings together with the above outlined advantages make the micromethod a suitable choice that may enable saliva sampling under certain circumstances.

\section{References}

[1] D. J. Adam, A. A. Milne, S. M. Evans, J. E. Roulston, A. J. Lee, C. V. Ruckley and A. W. Bradbury, "Serum Amylase Isoenzymes in Patients Undergoing Operation for Ruptured and Non-Ruptured Abdominal Aortic Aneurysm," Journal of Vascular Surgery, Vol. 30, No. 2, 1999, pp. 229-235. doi:10.1016/S0741-5214(99)70132-1

[2] P. M. Martinez, A. R. Torres, R. Ortiz de Lejarazu, A. Montoya, J. F. Martin and J. M. Eiros, "Human Immunodeficiency Virus Antibody Testing by Enzyme-Linked Fluorescent and Western Blot Assays Using Serum, Gingival-Crevicular Transudate, and Urine Samples," Journal of Clinical Microbiology, Vol. 37, No. 4, 1999, pp. 1100-1106.

[3] E. Filaire and G. Lac, "Dehydroepiandrosterone (DHEA) Rather than Testosterone Shows Saliva Androgen Re- 
sponses to Exercise in Elite Female Handball Players," International Journal of Sports Medicine, Vol. 21, No. 1, 2000, pp. 17-20. doi:10.1055/s-2000-8851

[4] S. P. Humphrey and R. T. Williamson, "A Review of Saliva: Normal Composition, Flow, and Function," Journal of Prosthetic Dentistry, Vol. 85, No. 2, 2001, pp. 162169. doi:10.1067/mpr.2001.113778

[5] C. F. Streckfus and L. R. Bigler, "Saliva as a Diagnostic Fluid," Oral Diseases, Vol. 8, No. 2, 2002, pp. 69-76. doi:10.1034/j.1601-0825.2002.10834.x

[6] D. B. Ferguson, "Current Diagnostic Uses of Saliva," Journal of Dental Research, Vol. 66, No. 2, 1987, pp. 420424. doi:10.1177/00220345870660020601

[7] D. Malamud, "Saliva as a Diagnostic Fluid," British Medical Journal, Vol. 305, No. 6847, 1992, pp. 207-208. doi:10.1136/bmj.305.6847.207

[8] I. D. Mandel, "A Contemporary View of Salivary Research," Critical Reviews in Oral Biology \& Medicine, Vol. 4, No. 3-4, 1993, pp. 599-604.

[9] H. C. Slavkin, "Toward Molecularly Based Diagnostics for the Oral Cavity," Journal of the American Dental Association, Vol. 129, No. 8, 1998, pp. 1138-1143.

[10] M. Navazesh, "Methods for Collecting Saliva," Annals of the New York Academy of Sciences, Vol. 20, No. 694, 1993, pp. 72-77. doi:10.1111/j.1749-6632.1993.tb18343.x

[11] N. Rohleder and U. M. Nater, "Determinants of Salivary Alpha-Amylase in Humans and Methodological Considerations," Psychoneuroendocrinology, Vol. 34, No. 4, 2009, pp. 469-485. doi:10.1016/j.psyneuen.2008.12.004

[12] E. A. Shirtcliff, D. A. Granger, E. Schwartz and M. J. Curran, "Use of Salivary Biomarkers in Biobehavioral Research: Cotton-Based Sample Collection Methods Can Interfere with Salivary Immunoassay Results," Psychoneuroendocrinology, Vol. 26, No. 2, 2001, pp. 165-173. doi:10.1016/S0306-4530(00)00042-1

[13] A. G. Harmon, L. C. Hibel, O. Rumyantseva and D. A. Granger, "Measuring Salivary Cortisol in Studies of Child Development: Watch out-What Goes in May Not Come out of Saliva Collection Devices," Developmental Psychobiology, Vol. 49, No. 5 2007, pp. 495-500. doi:10.1002/dev.20231

[14] J. A. DeCaro, "Methodological Considerations in the Use of Salivary a-Amylase as a Stress Marker in Field Research," American Journal of Human Biology, Vol. 20, No. 5, 2008, pp. 617-619. doi:10.1002/ajhb.20795

[15] J. A. Bosch, H. S. Brand, T. J. Ligtenberg, B. Bermond, J. Hoogstraten and A. V. Nieuw Amerongen, "Psychologi- cal Stress as a Determinant of Protein Levels," Psychosomatic Medicine, Vol. 58, No. 4, 1996, pp. 374-382.

[16] U. M. Nater, R. La Marca, L. Florin, A. Moses, W. Langhans, M. M. Koller and U. Ehlert, "Stress-Induced Changes in Human Salivary Alpha-Amylase ActivityAssociations with Adrenergic Activity," Psychoneuroendocrinology, Vol. 31, No. 1, 2006, pp. 49-58. doi:10.1016/j.psyneuen.2005.05.010

[17] D. A. Granger, K. T. Kivlighan, M. el-Sheikh, E. B. Gordis and L. R. Stroud, "Salivary Alpha-Amylase in Biobehavioral Research: Recent Developments and Applications," Annals of the New York Academy of Sciences, Vol. 1098, 2007, pp. 122-144. doi:10.1196/annals.1384.008

[18] M. Neu, M. Goldstein, D. Gao and M. L. Laudenslager, "Salivary Cortisol in Preterm Infants: Validation of a Simple Method for Collecting Saliva for Cortisol Determination," Early Human Development, Vol. 83, No. 1, 2007, pp. 47-54. doi:10.1016/j.earlhumdev.2006.04.003

[19] S. L. Udupa, A. R. Prabhakar and S. Tandon, "Alpha-Amylase Inhibitors in Foodstuffs," Food Chemistry, Vol. 34, No. 2, 1989, pp. 95-101. doi:10.1016/0308-8146(89)90077-0

[20] K. T. Kivlighan and D. A. Granger, "Salivary AlphaAmylase Response to Competition: Relation to Gender, Previous Experience, and Attitudes," Psychoneuroendocrinology, Vol. 31, No. 6, 2006, pp. 703-714. doi:10.1016/j.psyneuen.2006.01.007

[21] R. Nagler, S. Lischinsky, E. Diamond, N. Drigues, I. Klein and A. Z. Reznick, "Effect of Cigarette Smoke on Salivary Proteins and Enzyme Activities," Archives of Biochemistry and Biophysics, Vol. 379, No. 2, 2000, pp. 229-236. doi:10.1006/abbi.2000.1877

[22] E. S. Winn-Deen, H. David, G. Sigler and R. Chavez, "Development of a Direct Assay for Alpha-Amylase," Clinical Chemistry, Vol. 34, No. 10, 1988, pp. 2005-2008.

[23] A. Y. Foo and R. Bais, "Amylase Measurement with 2Chloro-4-Nitrophenyl Maltotrioside as Substrate," Clinica Chimica Acta, Vol. 272, No. 2, 1998, pp. 137-147. doi:10.1016/S0009-8981(98)00009-6

[24] H. Ben-Aryeh, M. Fisher, R. Szargel and D. Laufer, "Composition of Whole Unstimulated Saliva of Healthy Children: Changes with Age," Archives of Oral Biology, Vol. 35, No. 11, 1990, pp. 929-931. doi:10.1016/0003-9969(90)90075-L

[25] M. Kilian and B. Nyvad, "Ability to Bind Salivary Alpha-Amylase Discriminates Certain Viridans Group Streptococcal Species," Journal of Clinical Microbiology, Vol. 28, No. 11, 1990, pp. 2576-2577. 